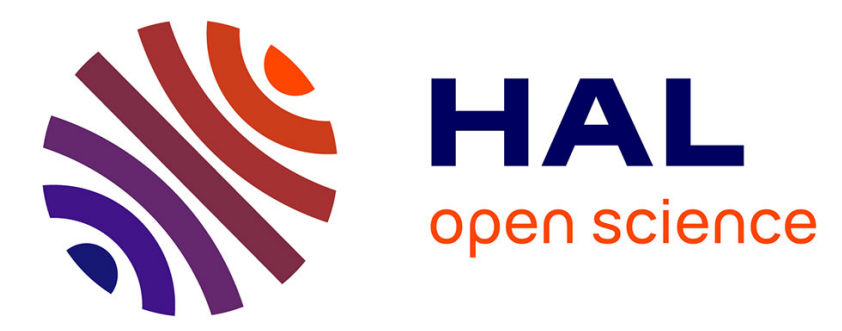

\title{
On the convectively unstable nature of optimal streaks in boundary layers
}

L. Brandt, Carlo Cossu, Jean-Marc Chomaz, Patrick Huerre, D.S. Henningson

\section{To cite this version:}

L. Brandt, Carlo Cossu, Jean-Marc Chomaz, Patrick Huerre, D.S. Henningson. On the convectively unstable nature of optimal streaks in boundary layers. Journal of Fluid Mechanics, 2003, may (485), pp.221-242. 10.1017/s0022112003004427 . hal-01024920

\section{HAL Id: hal-01024920 \\ https://hal-polytechnique.archives-ouvertes.fr/hal-01024920}

Submitted on 27 Aug 2014

HAL is a multi-disciplinary open access archive for the deposit and dissemination of scientific research documents, whether they are published or not. The documents may come from teaching and research institutions in France or abroad, or from public or private research centers.
L'archive ouverte pluridisciplinaire HAL, est destinée au dépôt et à la diffusion de documents scientifiques de niveau recherche, publiés ou non, émanant des établissements d'enseignement et de recherche français ou étrangers, des laboratoires publics ou privés. 


\title{
On the convectively unstable nature of optimal streaks in boundary layers
}

\author{
By LUCA BRANDT ${ }^{1}$, CARLO COSSU ${ }^{2}$, \\ JEAN-MARC CHOMAZ2, PATRICK HUERRE \\ AND DAN S. HENNINGSON ${ }^{1} \dagger$ \\ ${ }^{1}$ Department of Mechanics, Royal Institute of Technology (KTH), S-10044 Stockholm, Sweden \\ ${ }^{2}$ Laboratoire d'Hydrodynamique (LadHyX), CNRS-École Polytechnique, \\ F-91128 Palaiseau, France
}

(Received 9 April 2002 and in revised form 29 January 2003)

The objective of the study is to determine the absolute/convective nature of the secondary instability experienced by finite-amplitude streaks in the flat-plate boundary layer. A family of parallel streaky base flows is defined by extracting velocity profiles from direct numerical simulations of nonlinearly saturated optimal streaks. The computed impulse response of the streaky base flows is then determined as a function of streak amplitude and streamwise station. Both the temporal and spatio-temporal instability properties are directly retrieved from the impulse response wave packet, without solving the dispersion relation or applying the pinching point criterion in the complex wavenumber plane. The instability of optimal streaks is found to be unambiguously convective for all streak amplitudes and streamwise stations. It is more convective than the Blasius boundary layer in the absence of streaks; the trailing edge-velocity of a Tollmien-Schlichting wave packet in the Blasius boundary layer is around $35 \%$ of the free-stream velocity, while that of the wave packet riding on the streaky base flow is around $70 \%$. This is because the streak instability is primarily induced by the spanwise shear and the associated Reynolds stress production term is located further away from the wall, in a larger velocity region, than for the Tollmien-Schlichting instability. The streak impulse response consists of the sinuous mode of instability triggered by the spanwise wake-like profile, as confirmed by comparing the numerical results with the absolute/convective instability properties of the family of twodimensional wakes introduced by Monkewitz (1988). The convective nature of the secondary streak instability implies that the type of bypass transition studied here involves streaks that behave as amplifiers of external noise.

\section{Introduction}

It is now well established that the existence of a pocket of absolute instability plays a crucial role in accounting for the occurrence of synchronized self-sustained oscillations, or global modes, in a variety of spatially developing shear flows such as wakes, hot jets, counterflow mixing layers, etc. For a review of these concepts, see Huerre (2000). The theoretical and experimental studies of Lingwood $(1995,1996)$

$\dagger$ Also at FOI, The Swedish Defense Research Agency, Aeronautics Division, SE-17290 Stockholm, Sweden. 
have more recently demonstrated that in the case of the rotating disk boundary layer, a local change in the nature of the primary instability from convective to absolute surprisingly coincides with the transition from laminar flow to turbulence. In the same spirit as the latter transition studies, we would like to investigate the possibility that a related scenario might occur for the secondary instability of the nonlinearly saturated streaks which emerge from the spatial evolution of optimal perturbations in the flat-plate boundary layer. In other words, are the nonlinearly saturated streaks determined by Andersson et al. (2001) convectively unstable or absolutely unstable?

According to the theoretical and experimental studies of Gaster (1975) and Gaster \& Grant (1975), the primary Tollmien-Schlichting instability is unambiguously convective in nature; the impulse response of the Blasius boundary layer takes the form of a wave packet that is convected in the downstream direction. It is known, however, from the work of Butler \& Farrell (1992) among others, that the class of perturbations which sustain maximum temporal growth in the linear regime take the shape of spanwise periodic streamwise vortices. Such optimal perturbations give rise at maximum growth to a spanwise periodic distribution of low- and high-speed streaks. A similar conclusion holds if we consider instead optimal perturbations of maximum spatial growth, as demonstrated by Andersson, Berggren \& Henningson (1999) and Luchini (2000). A comprehensive presentation of hydrodynamic instability and transient growth phenomena in shear flows is given by Schmid \& Henningson (2001).

Spatial optimal perturbations (from a linear point of view) may be fed as upstream forcing into a direct numerical simulation of the flow along a flat plate, as carried out by Andersson et al. (2001). Optimal disturbances give rise to nonlinearly saturated streamwise streaks, which are spanwise periodic and therefore amenable to a secondary Floquet instability formulation, if the flow is assumed to be locally parallel. The main findings of this local inviscid secondary instability analysis are as follows: there exists a critical streak amplitude around $26 \%$ of the free-stream velocity, above which the streaky base flow becomes unstable to sinuous streamwise travelling waves. The most amplified perturbation is either a fundamental mode (with the same spanwise wavelength as the streaks) or a subharmonic mode (with twice the spanwise wavelength of the streaks), depending on the streak amplitude. The fundamental and subharmonic modes display quantitatively similar temporal instability characteristics in terms of both maximum growth rate and band of unstable streamwise wavenumbers. Streaks only become unstable to varicose streamwise travelling waves when their amplitude exceeds $37 \%$ of the free-stream velocity. The objective of our study is precisely to extend the temporal instability analysis of Andersson et al. (2001) by examining the spatio-temporal features of the impulse response. If a local absolute instability were found, we could relax the parallel flow assumption and look for self-sustained global modes in the spatially evolving boundary layer, as done by Pier \& Huerre (2001) for a two-dimensional wake. In fact, it is possible to show that for flows slowly varying in the streamwise direction, the existence of a pocket of absolute instability is a necessary condition for a global instability to occur (Chomaz, Huerre \& Redekopp 1991).

The investigations discussed above, as well as the present work, are motivated by the need to understand the physics of bypass transition in boundary layers with high levels of free-stream turbulence (Morkovin \& Reshokto 1990; Saric, Wu \& Choudari 2001; Reed \& Kerschen 2002). In this context, Matsubara \& Alfredsson (2001) have demonstrated experimentally that in boundary layers subject to free-stream turbulence, the appearance of streaks leads to unsteady oscillations and breakdown 
into turbulent spots. They review the recent literature on this topic. In order to identify the physical mechanisms responsible for breakdown, it appears essential to determine if the observed oscillations of streaks grow in situ, as in an absolute instability. If this were the case, we could pin down the onset of the oscillations and possibly of transition to the particular downstream station where the secondary instability shifts from convective to absolute. On the contrary, if the instability were to be convective, i.e. advected downstream, the development of the oscillations would depend strongly on the ambient upstream noise level. The perturbations considered here are steady and optimal only in the linear sense and therefore they represent one of several plausible approximations of the streaks observed in boundary layers subject to relatively high free-stream turbulence. However, it is important to note that in Matsubara \& Alfredsson (2001) the wall-normal shape of the linearly optimal disturbance theoretically determined by Andersson et al. (1999) and Luchini (2000) is found to be remarkably similar to the measured $u_{r m s}$ values. The streak can be regarded as a 'pseudo-mode' triggered in a boundary layer subject to significant outside disturbances.

The experiments of Swearingen \& Blackwelder (1987) were crucial in documenting the emergence of streaks with inflectional velocity profiles due to the formation of Görtler vortices in the boundary layer on a concave wall. This investigation demonstrated that the time-dependent fluctuations appearing in the flow are more closely correlated with the spanwise shear than with the wall-normal shear. The secondary linear instability of Görtler vortices was first analysed theoretically by Hall \& Horseman (1991). A generalized Rayleigh equation was derived for a class of three-dimensional base states consisting of the streak profiles generated by Görtler vortices. The fastest growing secondary mode was then found to be sinuous in character. Similar features prevailed for the secondary instability of temporallyevolving nonlinear Görtler vortices, as demonstrated theoretically by Park \& Huerre (1995). They confirmed that the dominant sinuous mode is primarily induced by the spanwise shear whereas the varicose mode is triggered by the wall-normal shear. The corresponding analysis of the secondary instability of streamwise-developing Görtler vortices was performed by Bottaro \& Klingmann (1996). This study led to a satisfactory prediction of the dominant frequency and sinuous perturbation velocity field observed by Swearingen \& Blackwelder (1987).

The widespread occurrence of streaks in various flow configurations has very recently led Asai, Minagawa \& Nishioka (2002) to examine experimentally the spatial response of a single low-speed streak in a laminar boundary layer submitted to a timeharmonic excitation of sinuous or varicose type. The growth of the sinuous mode was observed to evolve into a train of quasi-streamwise vortices with vorticity of alternate sign. By contrast, the varicose mode led to the formation of hairpin structures made up of a pair of counter-rotating vortices. Wu \& Luo (2001) proposed a new mechanism to explain the appearance of the varicose instability for low amplitudes of the streaks.

The possibility that in a given flow configuration, primary and secondary instabilities might display a distinct absolute/convective character was recognized by Huerre (1988) in the framework of a simple Ginzburg-Landau model equation. In many situations, the primary instability gives rise to spatially periodic saturated states. The secondary instability of such basic flows then typically requires the implementation of Floquet theory. The generalization of the classical absolute/convective instability analysis of Bers (1983) to the case of spatially periodic flows has been comprehensively carried out by Brevdo \& Bridges (1996). Their mathematical formulation provides in particular a precise convective/absolute instability criterion applicable to general 
spatially periodic flows. The implementation of such a pinching point criterion is, however, non-trivial and it has been applied only to model evolution equations, as in Brevdo \& Bridges (1996) and Chomaz, Couairon \& Julien (1999). For 'real' flows, the task of verifying the proper pinching of spatial branches appears formidable. Our approach will be radically different: we deliberately remain in physical space and retrieve the spatio-temporal instability characteristics a posteriori from direct numerical simulations of the linear impulse response. This strategy has been proved by Delbende, Chomaz \& Huerre (1998) in the context of the primary instability in swirling jets and wakes. The procedure yields results which compare extremely favourably with the more orthodox pinching point criterion, as applied in swirling jets and wakes by Olendraru et al. (1999). The impulse response method has also been successfully applied by Brancher \& Chomaz (1997) to examine secondary absolute/convective instabilities in a streamwise periodic array of Stuart vortices.

The present investigation is most closely related to the recent analysis of Koch (2002) on the absolute/convective nature of the secondary instability sustained by crossflow vortices in three-dimensional boundary layers. Koch determined the spatiotemporal characteristics of secondary fundamental instability modes, i.e. of the same wavelength as the crossflow vortices, in a direction perpendicular to the vortex axis. The implementation of a saddle-point continuation method in the complex wavenumber plane leads to the conclusion that the secondary instability is convective.

The paper is organized as follows. In $\S 2$, we summarize the main characteristics of the nonlinearly saturated streamwise-developing streaks determined in the numerical simulations of Andersson et al. (2001). Parallel streaks are then extracted from the simulations in order to define an appropriate family of base flows for the linear impulse calculations. The diagnostic tools which are essential in retrieving the temporal and spatio-temporal features of the secondary instability are presented, as well as the numerical methods necessary in the implementation of the impulse response simulations. The nature of the impulse response wave packet is analysed in $\S 3$. The temporal instability properties are first retrieved and compared with available inviscid dispersion relation calculations. The bulk of the section concentrates on the determination of the characteristic propagation velocities and growth rates of the wave packet as a function of streak amplitude and streamwise station. In the discussion of $\S 4$, we propose a physical explanation for the results in terms of the distribution of the Reynolds stress production term in a cross-stream plane. Finally, the crucial role of the spanwise shear in determining the propagation velocities of the wave packet is ascertained by comparison of our results with those pertaining to the family of two-dimensional wakes defined in Monkewitz (1988). The paper ends with a summary of the main conclusions.

\section{Flow configuration and spatio-temporal diagnostic tools}

\subsection{Base flow and physical configuration}

We consider the boundary layer over a flat plate and define the local Reynolds number, $R e=\left(U_{\infty} \delta_{*}\right) / \nu$, by means of the free-stream velocity $U_{\infty}$ and the local Blasius boundary-layer displacement thickness $\delta_{*}$. The analysis concerns the linear secondary instability of the streaks resulting from the nonlinear evolution of the spatial optimal perturbation in a zero-pressure-gradient boundary layer. This base flow was computed in Andersson et al. (2001) by solving the full Navier-Stokes equations. In that work, the complete velocity field representing the steady linear optimal perturbation calculated by Andersson et al. (1999) was used as input close to the 

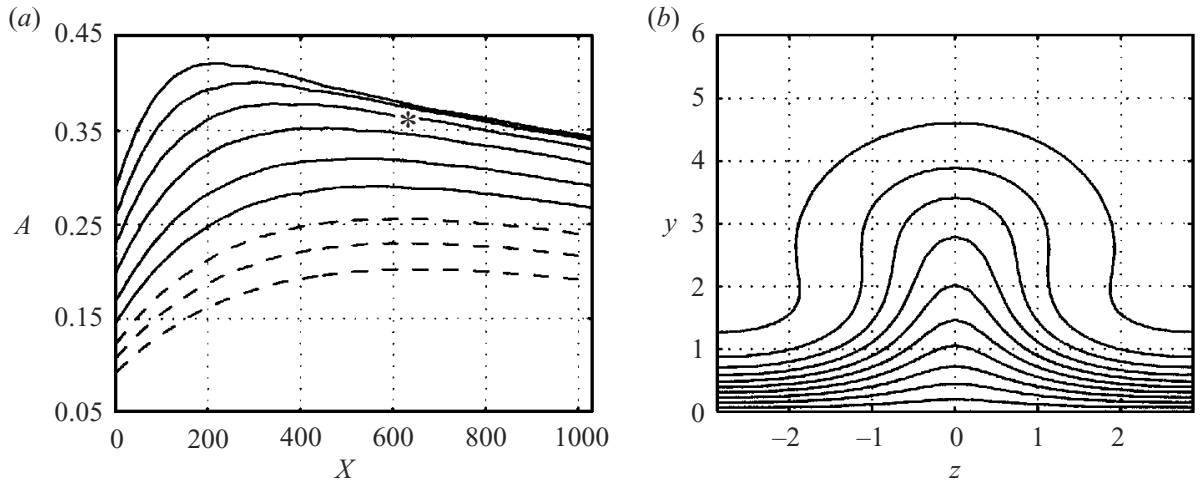

Figure 1. Base flow spatial simulations at $R e_{0}=468.4, \beta_{0}=0.48$. (a) Streak amplitude vs. streamwise coordinate $X$ for different upstream amplitudes $A=0.09,0.1,0.12,0.14,0.16$, $0.19,0.22,0.25,0.28$ at $X=0$. The three lower dashed curves correspond to stable streaks and the solid curves correspond to unstable streaks in some streamwise range. This plot reproduces figure 5(b) in Andersson et al. (2001). (b) Streamwise velocity contour plot of the nonlinear base flow in a $(y, z)$ cross-stream plane at $X=632, A=0.36, R e=1047$ for the condition indicated by an asterisk in $(a)$. Maximum contour level 0.98 , contour spacing 0.1 .

leading edge and its downstream nonlinear development was monitored for different upstream amplitudes of the input disturbance. The flow was assumed periodic in the spanwise direction and only one spanwise wavelength of the optimal perturbation considered. To quantify the size of this primary disturbance field at each streamwise position, an amplitude $A$ was defined in Andersson et al. (2001) as

$$
A(X)=\frac{1}{2}\left[\max _{y, z}\left(U(X, y, z)-U_{B}(X, y)\right)-\min _{y, z}\left(U(X, y, z)-U_{B}(X, y)\right)\right],
$$

where $U_{B}(X, y)$ is the Blasius profile and $U(X, y, z)$ is the total streamwise velocity in the presence of streaks. The streamwise velocity $U$ is made non-dimensional with respect to the free-stream velocity $U_{\infty}$. The streamwise distance $X$ is scaled with the inlet displacement thickness $\delta_{*_{0}}$ at the Reynolds number $R e=468.4$, i.e. at the inflow station $X=0$ in the spatial simulations of Andersson et al. (2001). The wall-normal and spanwise variables $y$ and $z$ are made non-dimensional with respect to the local Blasius boundary-layer displacement thickness $\delta_{*}(X)$. The spanwise wavenumber is taken to be $\beta_{0}=0.48$ at $X=0$, which corresponds to linearly optimally growing streaks at $X=246$. The downstream nonlinear amplitude development for different upstream amplitudes is displayed in figure $1(a)$, which reproduces figure $5(b)$ of Andersson et al. (2001). In figure 1(b), a typical nonlinearly saturated streak is illustrated by its streamwise velocity contour plot in the cross-stream $(y, z)$-plane. The selected streamwise station is $X=632$, which corresponds to $x / L=2$ in the scaling adopted in Andersson et al. (2001) where $L=1$ is the station of linear optimal growth, and the corresponding amplitude at this location is $A=0.36$. This condition, indicated by a star in figure 1(a), has been chosen because it is associated with the highest secondary instability temporal growth rate over all $X$ stations along that amplitude curve. As observed in Andersson et al. (2001), regions of strong spanwise shear are formed on the sides of the low-speed region, which is also displaced further away from the wall during the saturation process.

We are interested in determining the local spatio-temporal instability properties of the streaks in the parallel-flow approximation. In other words, we wish to study the 
local instability characteristics of a basic flow which evolves slowly in the streamwise direction, as required in the boundary-layer approximation. This assumption is justified since the instability is inviscid in nature and therefore leads to fast streamwise growth. A similar approach was followed in the case of Görtler vortices by Hall \& Horseman (1991) and Bottaro \& Klingmann (1996). The parallel-flow assumption was further validated in the direct numerical simulations of Andersson et al. (2001); time harmonic disturbances introduced upstream on the streaks were found to evolve with the spatial growth rate of the underlying parallel base flow, within a good degree of approximation.

In the present study, we therefore choose to perform numerical simulations of the linear impulse response for a basic parallel flow which consists only of the total streamwise velocity $U(y, z)$, the wall-normal and spanwise velocities of $O(1 / R e)$ being negligible. This basic parallel flow is extracted at different streamwise stations $X$ from the spatial numerical simulations of Andersson et al. (2001). In order to compensate for viscous diffusion, a body force $-\operatorname{Re}^{-1} \Delta U(y, z)$ is introduced in the numerical code so that the basic flow stays parallel and steady. The results of the local instability analysis are presented in terms of spatial and temporal variables $x, y, z$ and $t$ made non-dimensional with respect to the local boundary-layer displacement thickness $\delta_{*}$ and the free-stream velocity $U_{\infty}$.

According to classical Floquet theory (e.g. Nayfeh \& Mook 1979), for any flow variable $q$, instability modes of the spanwise periodic basic flow $U(y, z)$ of spanwise wavelength $\lambda_{z}$ may be sought in the form

$$
q(x, y, z, t)=\tilde{q}(y, z) \exp \left(\mathrm{i}\left(\left[k_{x} x+\delta k_{z} z-\omega t\right]\right)\right),
$$

where $\tilde{q}(y, z)$ is spanwise periodic and has the same wavelength $\lambda_{z}$ as $U(y, z), k_{x}$ is the streamwise wavenumber, $\omega$ is the circular frequency and $\delta k_{z}$ is the detuning parameter defined in the range $\left|\delta k_{z}\right| \leqslant \pi / \lambda_{z}$. In the present investigation, we restrict our attention to perturbations $q$ which have the same spanwise periodicity as the base flow, i.e. the detuning parameter is taken to be $\delta k_{z}=0$. This reduction to the fundamental mode is numerically implemented by considering a total flow (basic flow plus perturbation) which is spanwise periodic of fundamental wavelength $\lambda_{z}$. In this framework, the eigenfunctions of the system depend both on the spanwise $z$-variable and on the wall-normal $y$-variable. In the wall-normal direction, no-slip boundary conditions are imposed at the wall $y=0$ and zero velocity perturbation at $y=y_{\max }$, sufficiently high above the boundary layer.

To mimic forcing by a delta-function in time and space, the initial condition is defined in terms of a streamfunction as

$$
\psi(x, y, z ; 0)=A_{\epsilon} \bar{x} \bar{y}^{3} \bar{z} \exp \left(-\bar{x}^{2}-\bar{y}^{2}-\bar{z}^{2}\right),
$$

where $\bar{x}=\left(x-x_{0}\right) / l_{x}, \bar{y}=y / l_{y}$ and $\bar{z}=\left(z-z_{0}\right) / l_{z}$. The corresponding streamwise, wall-normal and spanwise velocity components are given by $(u, v, w)=\left(0, \psi_{z},-\psi_{y}\right)$ and the amplitude $A_{\epsilon}$ is set to sufficiently low values in order to ensure a linear development of the perturbations over a numerical integration run. This was checked by comparing the evolutions obtained for two different values of $A_{\epsilon}$. Typical values of the initial amplitude lie in the range $10^{-10}<A_{\epsilon}<10^{-6}$. The length scales, $l_{x}=5, l_{y}=2$ and $l_{z}=1.5$ have been chosen small enough to reproduce a localized impulse within the limits of a good resolution in the truncated spectral space of the numerical simulations. As an a posteriori test, it was verified that the initial condition contained all the physically relevant wavenumbers, typically twice the range of unstable streamwise wavenumbers. Note also that since the eigenfunctions 
depend on both $y$ and $z$, a strong localization is not required in these directions. Moreover, the disturbance is centred around $z=z_{0}$, which is chosen to lie off axis in one of the two regions of strongest spanwise shear (figure $1 b$ ) so that no particular symmetry is enforced on the solution. The initial condition (2.3) consists of two counter-rotating streamwise vortex pairs. It has been used previously by Henningson, Lundbladh \& Johansson (1993); Bech, Henningson \& Henkes (1998) and almost in the same form by Breuer \& Haritonidis (1990). Not only is the parallel flow spanwise periodic, which implies the Floquet decomposition (2.2), but also it is symmetric with respect to the $(x, y)$-plane. As a consequence, it admits two classes of normal modes: a sinuous mode with $u, v$ antisymmetric and $w$ symmetric in $z$, a varicose mode with $u, v$ symmetric and $w$ antisymmetric in $z$. As previously stated, the initial condition potentially excites these two types of disturbance.

\subsection{Diagnostics}

The technique developed by Delbende et al. (1998) and Delbende \& Chomaz (1998) is now extended to the case of a three-dimensional unidirectional base flow. This method allows us to retrieve the linear temporal and spatio-temporal instability features directly from the analysis of the simulated impulse response arising from the initial disturbance (2.3) described in the previous section. The analysis is thus performed in physical space according to the original definitions, without resorting to the spectral theory of absolute and convective instability. The procedure is only briefly outlined; for more details, the reader is referred to Delbende et al. (1998) and Delbende \& Chomaz (1998).

Within a temporal framework the wavenumber $k_{x}$ is given real and the frequency $\omega\left(k_{x}\right)$ is complex and unknown. Let us define the amplitude spectrum of the perturbations

$$
\tilde{e}\left(k_{x}, t\right)=\left(\int_{0}^{y_{\max }} \int_{0}^{\lambda_{z}}\left|\tilde{q}\left(k_{x}, y, z, t\right)\right|^{2} \mathrm{~d} y \mathrm{~d} z\right)^{1 / 2},
$$

where $q(x, y, z, t)$ may be any flow perturbation variable, e.g. a single velocity component or the square root of the total kinetic energy, and $\tilde{q}\left(k_{x}, y, z, t\right)$ its Fourier transform in the streamwise direction. For large times, the asymptotic exponential behaviour is attained and, if a well-separated temporal branch is present, the temporal growth rate $\omega_{i}$ of each $k_{x}$-component is given by

$$
\omega_{i}\left(k_{x}\right) \sim \frac{\partial}{\partial t} \ln \tilde{e}\left(k_{x}, t\right), \quad t \rightarrow \infty,
$$

where $\omega_{i}$ is the imaginary part of $\omega$. Recall here that, according to the temporal inviscid instability analysis presented in Andersson et al. (2001), only one unstable sinuous branch is expected to arise.

In the spatio-temporal formulation, the development of the wave packet along rays of specific given velocity $x / t=v$ is considered. This is equivalent to the investigation of modes of real group velocity $v$, as reviewed, for instance, in Huerre \& Rossi (1998) and Huerre (2000). In order to demodulate the wave packet and define its amplitude unambiguously, it is convenient, as in Delbende et al. (1998), to introduce the analytical complex field variable $\bar{q}(x, y, z, t)$ associated with $q(x, y, z, t)$ through the convolution

$$
\bar{q}(x, y, z, t)=\left[\delta(x)+\frac{\mathrm{i}}{\pi x}\right] * q(x, y, z, t),
$$


where the symbol $*$ designates the convolution operator with respect to $x$. The complex field $\bar{q}(x, y, z, t)$ in effect generalizes the complex exponential representation of a sine wave to an arbitrary real function $q(x, y, z, t)$ (Roddier 1971). In wavenumber space, equation (2.6) reduces to

$$
\bar{q}\left(k_{x}, y, z, t\right)=2 H\left(k_{x}\right) \tilde{q}\left(k_{x}, y, z, t\right),
$$

where $H\left(k_{x}\right)$ is the Heaviside unit-step function. In other words, the Fourier transform of the analytical field is obtained by setting to zero all the Fourier modes of negative streamwise wavenumber. As in the temporal analysis, the integration of the analytical field $\bar{q}$ in the cross-stream $(y, z)$-plane then yields the amplitude $Q$ defined by

$$
Q(x, t)=\left(\int_{0}^{y_{\max }} \int_{0}^{\lambda_{z}}|\bar{q}(x, y, z, t)|^{2} \mathrm{~d} y \mathrm{~d} z\right)^{1 / 2} .
$$

According to steepest-descent arguments (Bers 1983), the long-time behaviour of the wave packet along each spatio-temporal ray $x / t=v$ is

$$
Q(x, t) \propto t^{-1 / 2} \exp \left(\mathrm{i}\left(\left[k_{x}(v) x-\omega(v) t\right]\right)\right), \quad t \rightarrow \infty,
$$

where $k_{x}(v)$ and $\omega(v)$ represent the complex wavenumber and frequency travelling at the real group velocity $v=x / t$. For a detailed discussion of the meaning of group velocity for unstable systems, see Huerre \& Rossi (1998) among others. In (2.9), the real part of the exponential

$$
\sigma(v)=\omega_{i}(v)-k_{x, i}(v) v
$$

denotes the temporal growth rate observed while travelling at the group velocity $v$ and it can be evaluated for large $t$ directly from the amplitude $Q$ in (2.9) as

$$
\sigma(v) \sim \frac{\partial}{\partial t} \ln \left[t^{1 / 2} Q(v t, t)\right]
$$

In unstable flows, $\sigma>0$ for some range of $v$. The curves $\sigma(v)$ contain all the information characterizing the spatio-temporal growth of the wave packet. Its extent, in fact, is delineated by the rays along which neutral waves are observed. These velocities are denoted $v^{-}$, the trailing-edge velocity of the wave packet, and $v^{+}$, the leading-edge velocity of the wave packet, and they are formally defined by the conditions $\sigma\left(v^{-}\right)=\sigma\left(v^{+}\right)=0$ and $v^{-}<v^{+}$. In the present case, only one connected region of unstable group velocities is observed and the velocities $v^{-}$and $v^{+}$are unambiguously defined. If $v^{-}>0$, the wave packet is advected downstream and the base flow is convectively unstable. Conversely, if $v^{-}<0$, the trailing edge moves upstream and the wave packet grows in situ. The base flow is then absolutely unstable. Finally, let $v^{\max }$ denote the spatio-temporal ray along which the largest temporal growth rate $\sigma^{\max }$ is observed. This quantity provides a measure of the propagation velocity of the centre of the wave packet. According to Delbende et al. (1998), $\sigma^{\max }=\omega_{i}\left(k_{x}^{\max }\right)$, i.e. the maximum temporal amplification rate coincides with its maximum spatio-temporal counterpart, which is attained for a real wavenumber $k_{x}^{\max }$. This relation provides a further cross-check on the accuracy of the temporal and spatio-temporal analysis.

\subsection{Numerical methods}

The direct numerical simulations are performed with the code described in Lundbladh et al. (1999). Spectral methods are used to solve the three-dimensional time-dependent incompressible Navier-Stokes equations with the body force needed to remove the 
viscous diffusion of the base flow, as discussed in $\$ 2.1$. As in Delbende et al. (1998), we find it convenient to immerse the streamwise development of the wave packet in a streamwise periodic box which is sufficiently large so as to avoid 'recirculation effects'. Consequently, the computational domain is effectively periodic in both the $z-$ and $x$ directions. For the results presented here, streamwise lengths $L_{x}=600$ and $L_{x}=1200$ have been selected. A typical value of the box height is $y_{\max }=10$. The algorithm is similar to that of Kim, Moin \& Moser (1987), i.e. Fourier representation in the streamwise and spanwise directions and Chebyshev polynomials in the wall-normal direction, together with a pseudospectral treatment of the nonlinear terms. The time advancement used is a four-step low storage third-order Runge-Kutta scheme for the nonlinear terms and a second-order Crank-Nicholson method for the linear terms. Aliasing errors from the evaluation of the nonlinear terms are removed by the 3/2-rule when the fast Fourier transforms (FFTs) are calculated in the wall-parallel plane. In the wall-normal direction, it has been found more convenient to increase resolution rather than to use dealiasing. In all the results presented below, $n_{y}=97$ and $n_{z}=32$ modes have been chosen in the wall-normal and spanwise direction, respectively; in the streamwise direction $n_{x}=512$ or $n_{x}=1024$ modes have been deemed sufficient according to the length $L_{x}=600$ or $L_{x}=1200$ of the domain.

In order to evaluate the usual temporal growth rates and the temporal growth rates 'at the velocity $v$ ', equations (2.4) and (2.11) are numerically discretized (Delbende et al. 1998; Delbende \& Chomaz 1998) according to the expressions

$$
\begin{gathered}
\tilde{\omega}_{i}\left(k_{x}\right) \approx \frac{\ln \left[\tilde{e}\left(k_{x}, t 2\right) / \tilde{e}\left(k_{x}, t 1\right)\right]}{t_{2}-t_{1}}, \\
\sigma(v) \approx \frac{\ln \left[Q\left(v t_{2}, t_{2}\right) / Q\left(v t_{1}, t_{1}\right)\right]}{t_{2}-t_{1}}+\frac{\ln \left(t_{2} / t_{1}\right)}{2\left(t_{2}-t_{1}\right)} .
\end{gathered}
$$

In the above, $t_{1}$ and $t_{2}$ must be large enough so that transient effects are negligible. For each streak amplitude under consideration, different pairs $t_{1}, t_{2}$, are selected in the evaluation of (2.12) and (2.13) until results have satisfactorily converged. The values of $t_{1}, t_{2}$ change from case to case, with the least unstable modes requiring longer integration times and therefore longer streamwise distances. For the maximum temporal growth rates, a typical integration time is $t_{2}=300$ with $t_{2}-t_{1}=100$. For the lowest temporal growth rates, a typical integration time is $t_{2}=700$ with $t_{2}-t_{1}=200$. The analytical signal $\bar{q}(x, y, z, t)$ of $q(x, y, z, t)$ defined in (2.6) is computed in spectral space $\tilde{q}\left(k_{x}, y, z, t\right)$, where it reduces to setting to zero all the Fourier modes with negative wavenumber $k_{x}$. The uncertainty on all the results presented is at most $2 \%$.

\section{Streak linear impulse response}

The streak impulse response is displayed in physical space in figure 2 for the particular base flow of figure $1(b)$. The evolution in a plane parallel to the wall at a distance $y=1.8$ is illustrated. The background colour indicates the parallel base flow $U(y, z)$, with the low-speed region located at the centre of the computational domain. The blue contour lines represent the wall-normal velocity of the impulse response wave packet at three different times $t=0, t=200$ and $t=400$, so that the initial condition, defined by equation (2.3), can be readily seen. The emerging wave packet is sinuous with respect to the background streak, since its wall-normal velocity is antisymetric in $z$. According to the temporal analysis of Andersson et al. (2001), the 

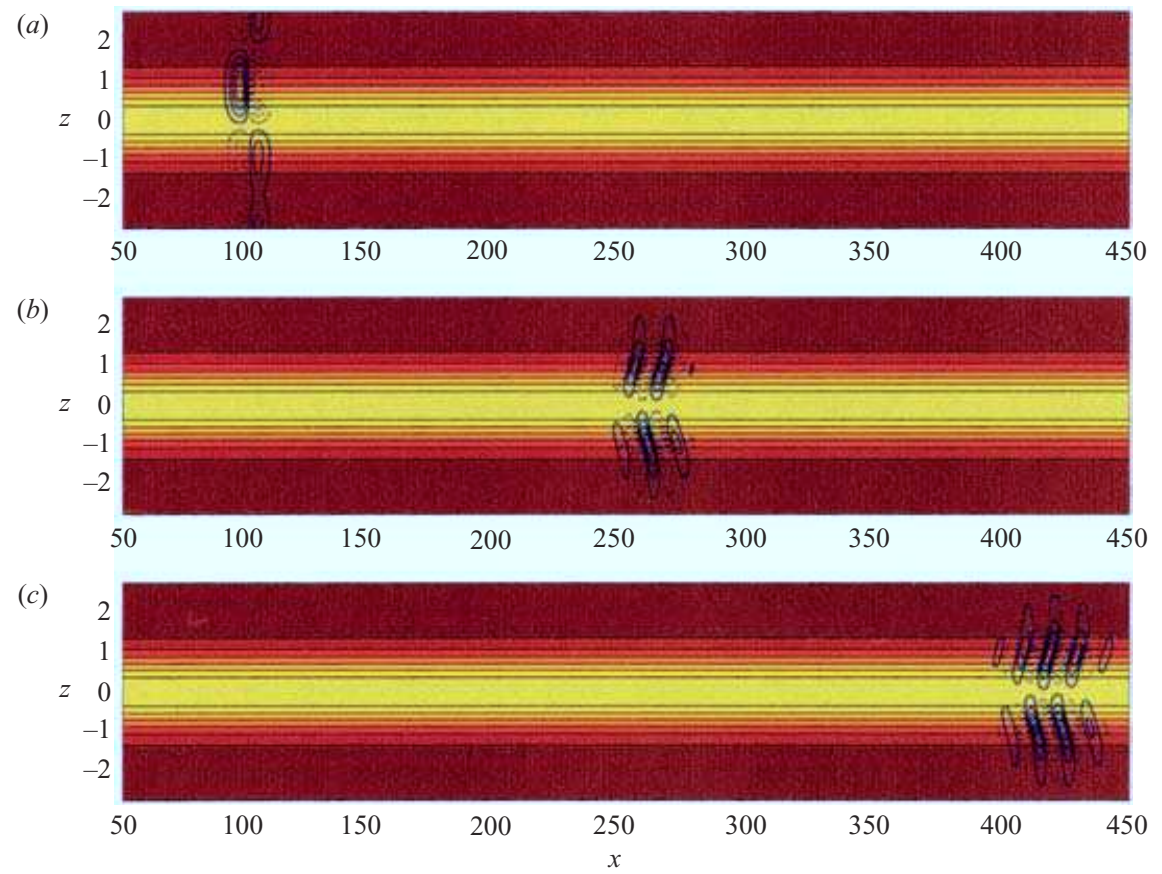

FIGURE 2. Streak impulse response of the base flow defined in figure $1(b)$ in a plane parallel to the wall, at $y=1.8$, for $(a) t=0,(b) t=200$ and $(c) t=400$. The initial packet is centred around $z_{0}=0.85$. The background colour represent the streamwise velocity of the base flow, with high to lower velocity ranging from red to yellow. The blue isolines indicate the wall-normal perturbation velocity; dotted lines denote negative values.

sinuous mode was indeed the only one found to be unstable for this particular streak. Note furthermore that the $v$-structures of the packet are bent in the downstream direction in the outer parts where the basic advection is larger. The downstream propagation of the wave packet appears to indicate that the streak instability is convective. This conclusion, however, is premature; the trailing-edge velocity $v^{-}$of the wave packet must be unambiguously determined. Figure 2 merely demonstrates that the crest of the wave packet is advected downstream.

\subsection{Temporal instability}

Temporal instability results are presented with the aim of comparing them to the inviscid analysis of Andersson et al. (2001) so that the present formulation may be validated. The amplitude spectrum $\tilde{e}\left(k_{x}, t\right)$ is extracted from the impulse response simulations by means of (2.4) and equation (2.12) is then used to retrieve the temporal growth rate $\omega_{i}$ for different streamwise wavenumbers. The results for the base flow depicted in figure $1(b)$ are displayed in figure 3 for different values of the Reynolds number and they are compared with the inviscid instability results of Andersson et al. (2001) obtained directly from the dispersion relation. The latter calculation is reproduced as a thick solid line, whereas the thin solid line represents the growth rate curve at $R e=1047$, i.e. the local Reynolds number prevailing at the streamwise station indicated by an asterisk in figure $1(a)$. Since a streak family $U(X, y, z)$, defined by the upstream amplitude $A_{0}$ and the spanwise wavenumber $\beta_{0}$, obeys the nonlinear boundary-layer equations, it is independent of the Reynolds number (Schlichting 1979; Andersson et al. 2001). As a consequence, varying the local Reynolds number 


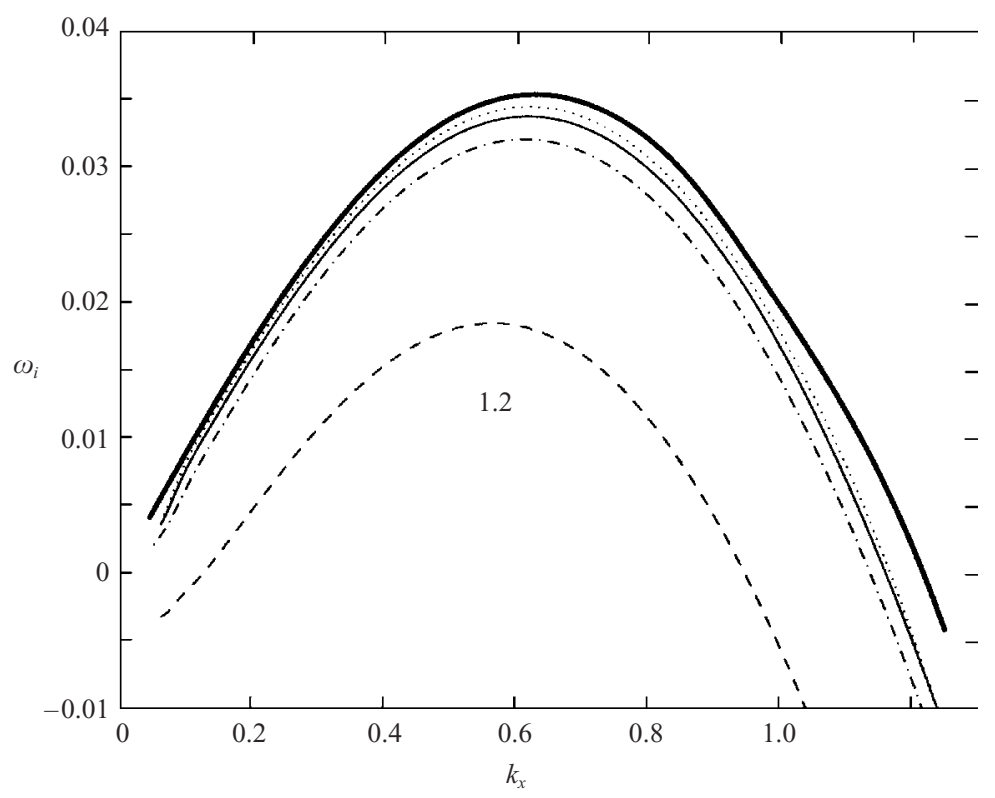

FiguRE 3. Temporal growth rate $\omega_{i}$ versus streamwise wavenumber $k_{x}$ for the base flow of figure 1(b). The inviscid results of Andersson et al. (2001) are represented by the thick solid line: $-; R e=1047:-; R e=2000: \cdots ; R e=500:-\cdot-; R e=100$ : - - - .

$\operatorname{Re}(X)$ of a given profile $U(y, z)$ amounts to moving along the plate and varying the spanwise wavenumber $\beta_{0}$ so that the local spanwise wavenumber $\beta_{0} \delta_{*} / \delta_{*_{0}}$ remains constant. The impulse response corresponding to such base flows yields in figure 3 the various growth rate curves indicated by a dotted line $(R e=2000)$, a dash-dotted line $(R e=500)$ and a dashed line $(R e=100)$. As expected, we note that the viscous temporal growth rate curves approach their inviscid counterpart as the Reynolds number increases, which validates the present approach. Furthermore, the range of unstable wavenumbers shrinks as viscosity is enhanced and the most unstable wavenumber $k_{x}^{\max }$ decreases. Higher wavenumbers are noticeably more affected by viscosity than lower wavenumbers.

In figure 3, we have avoided displaying results at very low wavenumbers since two kinds of difficulty are encountered. First, the impulse response approach is intrinsically limited to streamwise wavelengths smaller than the length of the computational domain. A further restrictive factor is related to the fact that the full nonlinear NavierStokes equations are effectively solved in the numerical code. As a consequence, the long-lived transients at low $k_{x}$ are contaminated by nonlinear interactions from higher wavenumbers before a reliable exponential growth rate becomes observable. Since the primary goal of this investigation remains the determination of the spatio-temporal behaviour, curing this problem has not been pursued further. In any case, the parallel flow assumption breaks down at long wavelengths.

\subsection{Spatio-temporal instability}

To determine the spatio-temporal instability properties of the streaks, the wave packet velocity field is observed in physical space along rays of group velocity $x / t=v$, as outlined in $\S 2.2$. The evolution of the amplitude $Q(x, t)$, defined by equation (2.8), is represented in figure 4 as a function of $v$ at different times, again for the base flow of figure $1(b)$. Note that the amplitude grows between two well-defined trailing- and 


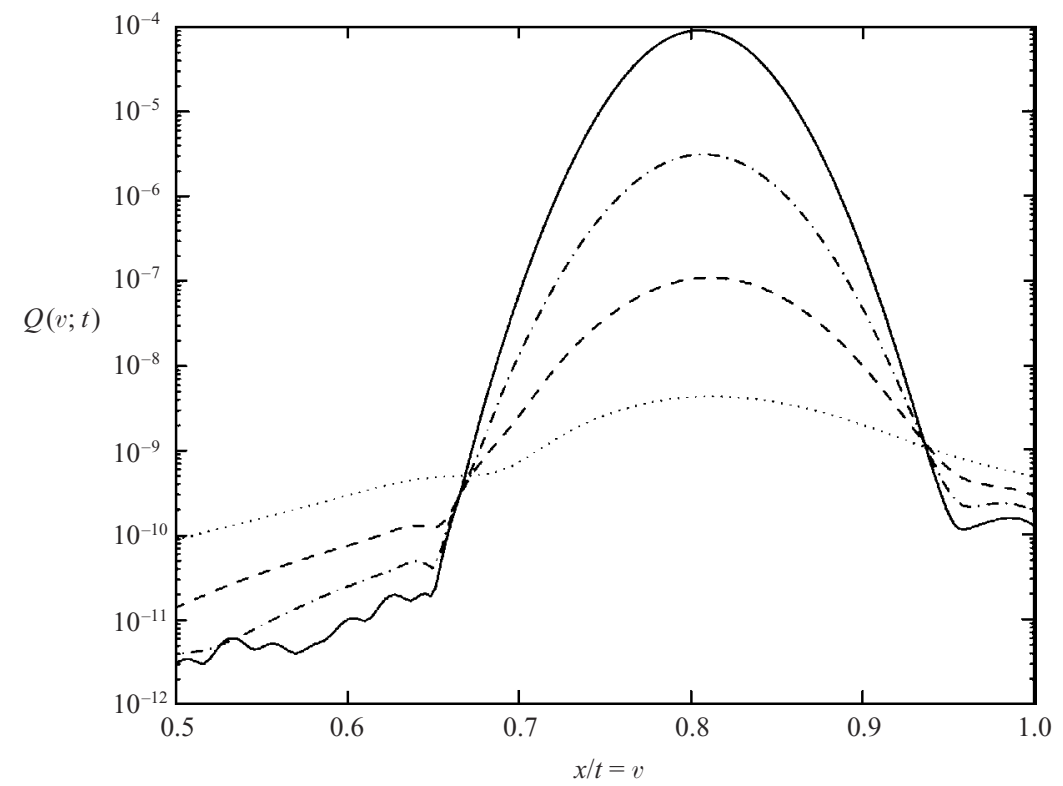

Figure 4. Amplitude $Q$ of the wave packet as a function of the ray velocity $x / t=v$ for different times $t=100: \cdots ; t=200:--; t=300:-\cdot-; t=400:-$. The base flow is extracted at $X=632, A=0.36, R e=1047$ as in figure $1(b)$.

leading-edge velocities $v^{-}$and $v^{+}$. The first neutral ray $x / t=v^{-}$is positive and therefore it may immediately be concluded that the instability is convective. It is also worth noting that the amplitude of the wave packet ahead of the velocity $v^{+}\left(v>v^{+}\right)$ experiences lower decay rates than the tail of the wave packet behind the velocity $v^{-}$ $\left(v<v^{-}\right)$. Since the front part is associated to velocities of order unity, it corresponds to the contribution from the continuous spectrum of disturbances (Grosch \& Salwen $1978)$, i.e. free-stream perturbations that are advected at the free-stream velocity and damped by viscous diffusion. The growth rate $\sigma(v)$ obtained from the data in figure 4 by applying equation (2.13) is displayed by the solid line in figure 5 . The growth rate curve is close to a parabola with trailing- and leading-edge velocities $v^{-}=0.66$ and $v^{+}=0.935$. The maximum growth rate is $\sigma^{\max }=0.0338$ and it is attained along the ray $v^{\max }=0.80$. The maximum temporal growth rate was found to be $\omega_{i}^{\max }=0.0337$ (see figure 3 ). The agreement, within $1 \%$, between these two numerically determined values constitutes a further validation check of the impulse response approach.

The temporal growth rate curve versus group velocity is displayed in figure 5 for other unstable streak amplitudes at streamwise position $X=632$. Such base states correspond to lower and higher amplitudes $A$ along the vertical line $X=632$ in figure $1(a)$. This growth rate diagram constitutes the spatio-temporal analogue of the inviscid temporal growth rates curves (figure $16 a$ in Andersson et al. 2001) for the same streak amplitude settings at the same streamwise station. Recall that, in this earlier study, the critical streak amplitude for breakdown was determined and the most dangerous type of disturbance was identified to be the sinuous mode of instability. It can be seen from figure 5 that the growth rates and the spreading rate $\Delta v \equiv v^{+}-v^{-}$of the wave packet increase with streak amplitude. Moreover, the maximum growth rate $\sigma^{\max }$ occurs approximately at the group velocity $v^{\max }=0.8$ for all streak amplitudes. 


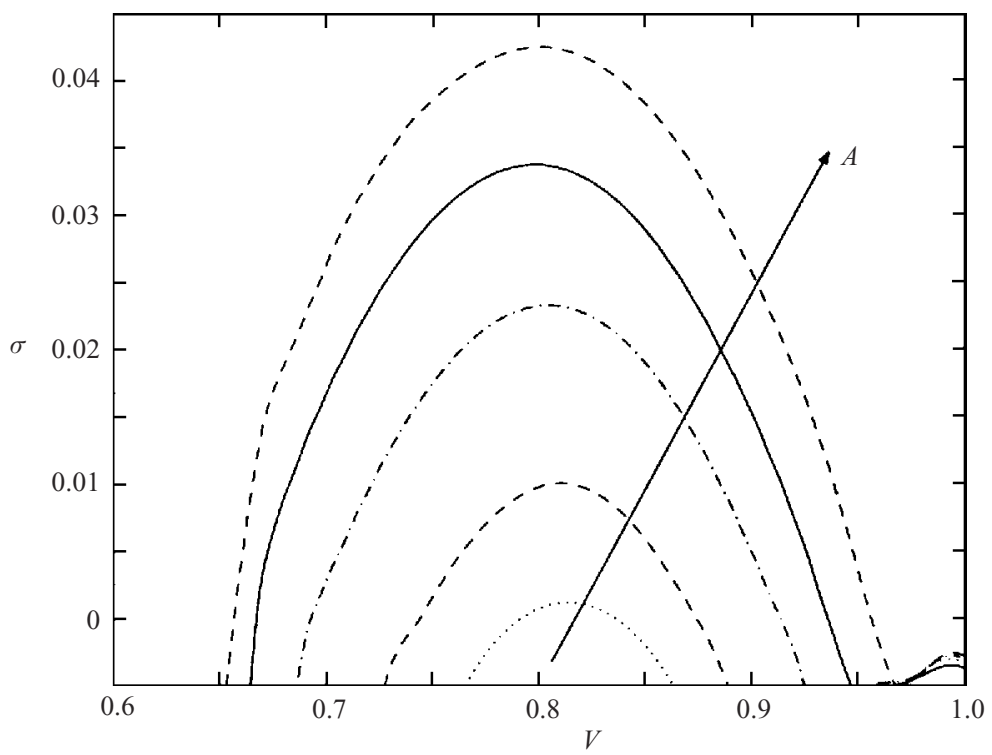

FIGURE 5. Temporal growth rate versus ray velocity $x / t=v$ for different increasing saturated streak amplitudes $A=0.28,0.31,0.34,0.36$ and 0.38 at $X=632$ and $R e=1047$. The solid line corresponds to the base flow at $A=0.36$ depicted in figure $1(b)$ and indicated by an asterisk in figure $1(a)$. The other curves correspond to scanning the vertical line $X=632$ of unstable streaks in figure 1(a) for lower and higher amplitude settings.

This value is quite close to the phase velocities $c_{r}=\omega_{r} / k_{x}$ obtained in the temporal analysis of Andersson et al. (2001), as displayed in figure 16(b) of that paper.

A parametric study has been carried out to fully document the main features of the impulse response for different amplitudes and streamwise positions. The results are synthesized in figure 6 . The characteristic velocities $v^{-}, v^{+}$and $v^{\max }$ are displayed in figure $6(a)$, while the maximum growth rate $\sigma^{\max }$ is shown in figure $6(b)$. The data are plotted as a function of the streamwise coordinate $X$ for three distinct streak evolution curves corresponding to upstream amplitudes $A=0.28,0.22$ and 0.16 at $X=0$ in figure $1(a)$. These upstream settings give rise to the first, third and fifth highest streak evolution curves in figure $1(a)$. Note that for the lowest amplitude considered (dashdotted lines in figure 6), the streak becomes unstable only for $X>450$. It can first be seen that $v^{\max }$ is quite insensitive to streamwise position and streak amplitude. Furthermore, the spreading rate of the wave packet $\Delta v=v^{+}-v^{-}$increases together with the maximum temporal growth rate $\sigma^{\max }$. For the highest amplitude streak (solid lines in figure 6) the growth rate $\sigma^{\max }$ reaches a maximum for small $X$ while the wave packet centre velocity is slightly lower than further downstream.

From the above parametric study, it can be concluded that the impulse response of streaks gives rise to a wave packet with a centre velocity $v^{\max }$ of the order of $80 \%$ of the free-stream velocity $U_{\infty}$. The wave packet spreading rate varies with the streak amplitude and the position $X$ between approximately $10 \%$ and $30 \%$ of $U_{\infty}$; the leading edge moves at a velocity $v^{+}$which is around $90 \%$ of $U_{\infty}$ while the trailing edge moves at a velocity $v^{-}$which is around $70 \%$ of $U_{\infty}$. According to figure 6 , the latter estimates are quite sensitive to streak amplitude. In any case, we may unambiguously state that the secondary instability of optimal streaks is highly convective for a wide range of amplitudes and streamwise stations. 

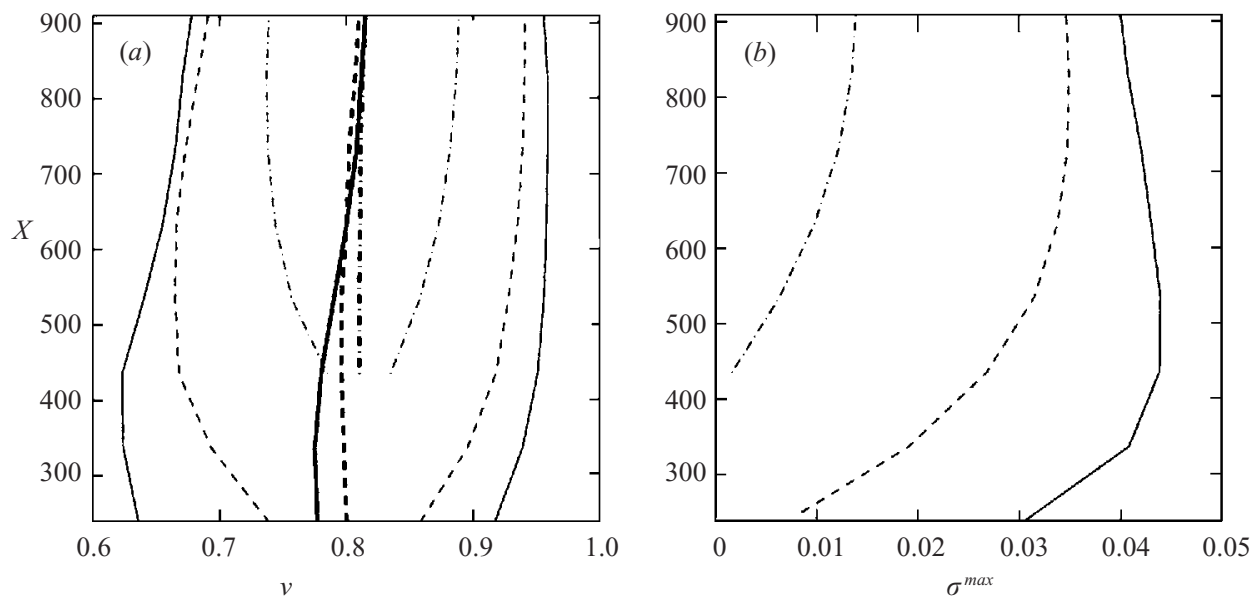

FIGURE 6. Impulse response characteristics of extracted parallel streaks as a function of streamwise distance $X$ for three distinct upstream streak amplitudes at $X=0 . A=0.28$ : - ; $A=0.22:---; A=0.16: \cdots(a)$ Leading-edge velocity $v^{+}$(thin lines), trailing-edge velocity $v^{-}$(thin lines), centre velocity $v^{\max }$ (thick lines). (b) Maximum temporal growth rate $\sigma^{\max }$.

\section{Discussion}

A feature of the above results is that the secondary streak instability is 'even more convective' than the primary Tollmien-Schlichting instability of the Blasius velocity profile. According to Gaster (1975) and Gaster \& Grant (1975), the leading-edge and trailing-edge velocities of a Tollmien-Schlichting wave packet in the absence of streaks are, respectively, $v^{+}=0.5$ and $v^{-}=0.36$ at $R e=1000$. In the presence of streaks, the trailing-edge velocity is $v^{-} \sim 0.7$. Thus, unstable perturbations travel much faster on the streaky base flow. Surely, these two situations correspond to radically distinct instability mechanisms: while the Tollmien-Schlichting instability is driven by viscosity, the streak instability is essentially inviscid in nature and driven by the presence of inflection points in the velocity profile. In order to further examine this behaviour, it is useful to evaluate, for both instances, the distribution of the kinetic energy production terms in the cross-stream $(y, z)$-plane.

Consider the perturbation kinetic energy equation averaged over the streamwise length $L_{x}$ of the box

$$
\begin{aligned}
\frac{\mathrm{d}}{\mathrm{d} t}\left[\int _ { 0 } ^ { y _ { \operatorname { m a x } } } \int _ { 0 } ^ { \lambda _ { z } } \frac { 1 } { 2 } \left(\overline{u^{2}}\right.\right. & \left.\left.+\overline{v^{2}}+\overline{w^{2}}\right) \mathrm{~d} y \mathrm{~d} z\right]=-\int_{0}^{y_{\max }} \int_{0}^{\lambda_{z}} \frac{\partial U}{\partial y} \overline{u v} \mathrm{~d} y \mathrm{~d} z \\
& -\int_{0}^{y_{\max }} \int_{0}^{\lambda_{z}} \frac{\partial U}{\partial z} \overline{u w} \mathrm{~d} y \mathrm{~d} z-\frac{1}{\operatorname{Re}} \int_{0}^{y_{\max }} \int_{0}^{\lambda_{z}} \overline{\boldsymbol{\omega} \cdot \boldsymbol{\omega}} \mathrm{d} y \mathrm{~d} z
\end{aligned}
$$

where a bar denotes the streamwise average

$$
\overline{(\cdot)}=\frac{1}{L_{x}} \int_{0}^{L_{x}}(\cdot) \mathrm{d} x,
$$

and $\omega$ the perturbation vorticity vector. This balance equation is derived in a straightforward manner from the Navier-Stokes equations linearized around the base flow $U(y, z)$. The first production term of density $-(\partial U / \partial y) \overline{u v}$ represents the work of the Reynolds stress $\tau_{x y}=-\overline{u v}$ on the wall-normal basic shear $(\partial U / \partial y)$, while the second production term of density $-(\partial U / \partial z) \overline{u w}$ is associated with the work 

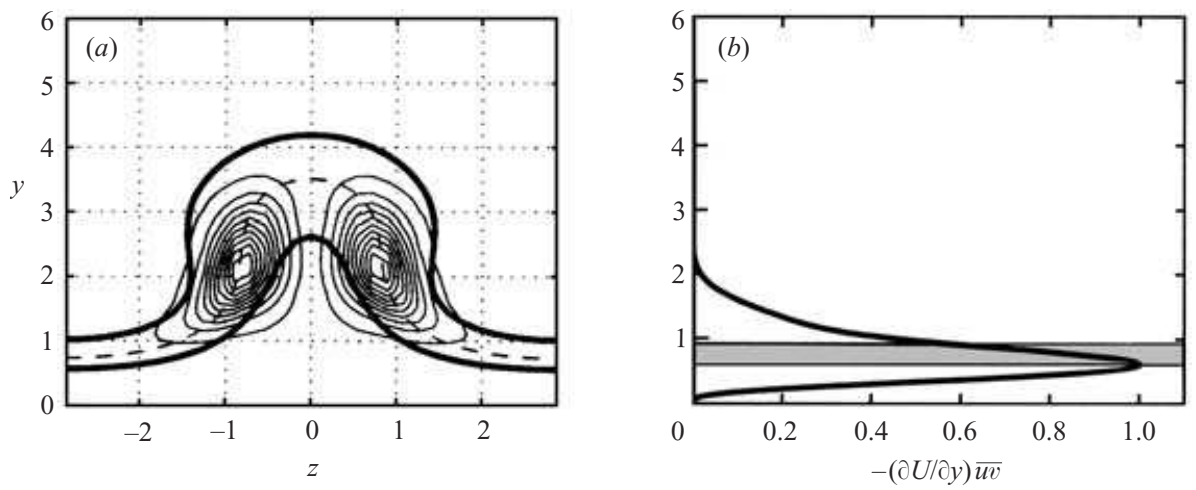

FIGURE 7. Production of perturbation kinetic energy and wave packet characteristic velocities. (a) Contour levels (thin solid lines) of the total production density $-(\partial U / \partial y) \overline{u v}-(\partial U / \partial z) \overline{u w}$ in a cross-stream $(y, z)$-plane for the impulse response of the streaky base flow of figure $1(b)$. The outer contour is $5 \%$ of the maximum and contour spacing is $10 \%$ of the maximum. The thick solid lines represent the base flow velocity contours $U(y, z)=v^{+}$and $U(y, z)=v^{-}$ with $v^{+}=0.935$ and $v^{-}=0.66$. The dashed line represents the base flow velocity contour $U(y, z)=v^{\max }$ with $v^{\max }=0.80$. (b) Wall-normal profile (thick solid line) of the production density $-(\partial U / \partial y) \overline{u v}$ for the impulse response of the Blasius base flow. The grey horizontal strip delineates the region of base flow velocities $v^{-}<U_{B}(y)<v^{+}$with $v^{+}=0.5$ and $v^{-}=0.36$.

of the Reynolds stress $\tau_{x z}=-\overline{u w}$ on the spanwise basic shear $\partial U / \partial z$. The last term represents viscous dissipation. Contour levels of the total production density $-(\partial U / \partial y) \overline{u v}-(\partial U / \partial z) \overline{u w}$ are displayed in figure $7(a)$ for the streaky base flow of figure $1(a)$, at time $t=300$, together with the contours $U(y, z)=v^{+}, U(y, z)=v^{-}$ and $U(y, z)=v^{\max }$ pertaining to the wave packet. For comparison, we have displayed in figure $7(b)$ the wall-normal profile of the production density term $-(\partial U / \partial y) \overline{u v}$ corresponding to the two-dimensional impulse response of the primary TollmienSchlichting instability in the Blasius boundary layer at the same Reynolds number $R e=1047$. In this case, there is no spanwise-shear production term since there are no spanwise variations. The grey horizontal strip delineates the region $v^{-}<U_{B}(y)<v^{+}$. The results of Gaster (1975) are recovered for the leading- and trailing-edge velocities.

In the case of streaks (figure $7 a$ ), the wall-normal shear production term $-(\partial U / \partial y) \overline{u v}$ is found to be negative and one order of magnitude smaller than the spanwise shear production term $-(\partial U / \partial z) \overline{u w}$. The streak instability is of inviscid inflectional type and primarily triggered by the regions of high spanwise shear on the flanks of the low-speed region. The areas of strongest production lie slightly above the position of maximum shear at $y=1.5$ in figure $7(a)$. The regions of largest energy production density are well correlated not only with the areas of high spanwise shear but also with the regions of streamwise velocity fluctuations (see Bottaro \& Klingmann 1996). This finding is in qualitative agreement with earlier investigations of streamwise vortices; according to Swearingen \& Blackwelder (1987), the experimentally observed sinuous instability of Görtler vortices is primarily associated with the spanwise shear of the streaky flow. Park \& Huerre (1995) later established theoretically that the sinuous instability was indeed driven by the spanwise shear production term. According to figure $7(a)$, the basic streamwise velocity prevailing at the point of maximum production is seen to coincide with the centre velocity $v^{\max }$ of the wave packet. In the same way, the regions of significant production are seen to be approximately confined in a domain where the basic streamwise velocity is such that 
$v^{-}<U(y, z)<v^{+}$. These features reveal the strong correlation between the basic velocities in the production area and the characteristic velocities of the wave packet.

In the case of the Blasius boundary layer (figure $7 b$ ), the wall-normal shear production term is confined in a region closer to the wall, where the basic velocities are smaller. The correlation between production location and the band $v^{-}<U_{B}(y)<v^{+}$ is not as obvious quantitatively. Nonetheless, the fact that $v^{-}$and $v^{+}$are smaller than in the case of streaks is qualitatively consistent with the location of production closer to the wall. (In general, there is no reason to expect that the border of the production region should coincide with the contours $U(y, z)=v^{+}$and $U(y, z)=v^{-}$.)

It should be emphasized again that the impulse response properties are very much dictated by the nature of the instability mechanisms. In the case of the Blasius layer, the mechanism is viscous and active in low basic velocity areas close to the wall, whereas in the case of streaks, it is inviscid in nature and active in higher basic velocity areas further away from the wall. As a result, the impulse response wave packet of streaks travels faster downstream than its counterpart for the Blasius profile. These features account for the fact that the presence of streaks makes the flow even more convectively unstable than the Blasius layer.

It might be argued that the Tollmien-Schlichting instability is still active when streaks are present, even if the associated growth rates are much smaller than those of the inviscid sinuous instability. Cossu \& Brandt (2002) have demonstrated that Tollmien-Schlichting waves do remain unstable for streak amplitudes that are smaller than any of those considered here. However, Tollmien-Schlichting waves are quenched above a certain streak amplitude level which is Reynolds-number dependent. In the range of Reynolds numbers considered here, this level is about $17 \%$ of $U_{\infty}$ and lower than the threshold for the onset of the streak instability, which is $26 \%$ of $U_{\infty}$.

The sinuous streak instability is induced by the mean spanwise shear on the sides of the region of defect velocity as in classical two-dimensional wakes past a bluff body. It is therefore instructive to compare the instability properties of the streaky base flows with those of two-dimensional wakes (LeCunff \& Bottaro 1993). At this point, we may note that for the bluff body, the wake and the resulting instability are isolated in the spanwise direction, whereas the streak instability arises on a spanwise periodic basic flow. However, the production density spatial distribution represented in figure 7(a) indicates that the streak instability is triggered locally by the basic spanwise shear. This is further confirmed by the complete Floquet analysis in Andersson et al. (2001) which demonstrates that the instability features are insensitive to variations in the Floquet spanwise detuning parameter.

To carry out the comparison, let us consider the symmetric wake profiles introduced by Monkewitz (1988) and defined by the two-parameter family

$$
U_{w}\left(z_{w} ; R, N\right)=1-R+2 R F\left(z_{w} ; N\right),
$$

where

and

$$
R \equiv \frac{U_{c}-U_{\max }}{U_{c}+U_{\max }}
$$

$$
F\left(z_{w} ; N\right) \equiv \frac{1}{1+\sinh ^{2 N}\left[z_{w} \sinh ^{-1}(1)\right]} .
$$

In the above relations, the length scale is the half-width of the wake and the velocity scale is the average velocity $\left(U_{c}+U_{\max }\right) / 2$, where $U_{c}$ is the minimum velocity on the centreline and $U_{\max }$ the maximum velocity in the high-speed regions. The velocity ratio 


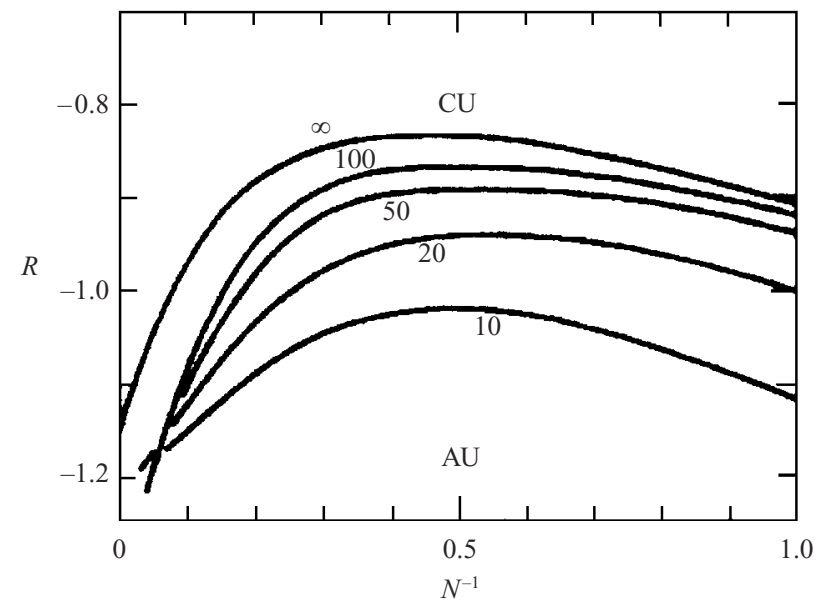

Figure 8. Absolute/convective instability boundary in the $\left(N^{-1}, R\right)$-plane at various wake Reynolds numbers indicated near the curves for the wake profile family (4.2). AU and CU denote the domain of absolute and convective instability, respectively. After Monkewitz (1988).

$R$ controls the depth of the wake. The shape factor $N$, which controls the steepness of the shear layers, varies between one, corresponding to the fully developed $\operatorname{sech}^{2} z$ wake, to infinity, a top-hat wake bounded by two vortex sheets. The curves representing the absolute instability boundary in the $R-N^{-1}$ plane for different Reynolds numbers are reproduced from Monkewitz (1988) in figure 8. In order to match our streak base state $U(y, z)$ with the family of wakes $U_{w}\left(z_{w}\right)$, we must select a spanwise profile at a specific wall-normal distance and extend it for all $y$ so as to obtain a corresponding $y$ invariant wake. Let us choose the spanwise streak profile at the wall-normal distance $y=2.1$ displayed as a solid line in figure 9 and associated with the Reynolds stress production peak in figure $7(a)$. Note that this profile is typical of fully developed streaks induced by free-stream turbulence. It may differ in detail from those produced by well-controlled artificial disturbances close to the excitation station. For instance, Asai et al. (2002) observe velocity overshoots on both sides of the wake profile which are smoothed out further downstream of the source region.

The best fit to the chosen profile with Monkewitz' family, indicated by a dotted line in figure 9 , is obtained for $N=1$ and $R=-0.25$, provided that $z_{w}=0.8 z$. Such a scale factor applied to the spanwise variable effectively means that the streak half-width is $80 \%$ of the local boundary-layer displacement thickness. The wake Reynolds number defined with respect to its half-width and the average velocity $\left(U_{c}+U_{\max }\right) / 2$ is then found to be 960 , instead of 1047 in boundary-layer variables. According to Monkewitz (1988), inviscid results are applicable as soon as the wake Reynolds number exceeds 200; the relevant absolute/convective instability transition curve in figure 8 therefore pertains to $R e=\infty$. The fitted wake $N=1, R=-0.25$ is seen to lie above the range of $R$-values in figure 8 on the convectively unstable side. It is clearly well above the absolute/convective instability transition curve at $R e=\infty$. If this fitting procedure is repeated at other wall-normal distances in the region of significant spanwise shear, we find that the velocity ratio varies within the range $-0.35<R<-0.2$, which is again too high for absolute instability to occur.

The above procedure may be further validated by inferring from Monkewitz' results the trailing-edge velocity $v^{-}$of the streak impulse response. By definition, the trailing-edge velocity is such that, in the co-moving frame, the instability is marginally 


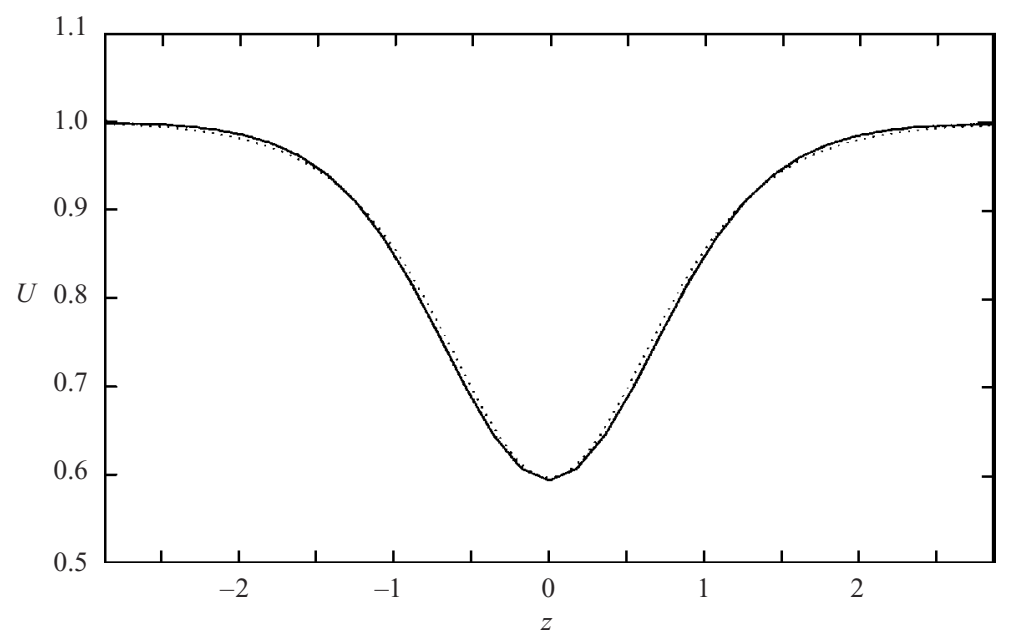

FIGURE 9. Wake-like velocity profile in the spanwise direction $z$. Base flow of figure $1(b)$ at the maximum production elevation $y=2.1$ : $\longrightarrow$; Monkewitz (1988) model of two-dimensional wakes with $N=1, R=-0.25$ and $z_{w}=0.8 z: \cdots$.

convective/absolute at the velocity ratio $R_{c r}$. In other words, $v^{-}$is given by

$$
\frac{\left(U_{c}-v^{-}\right)-\left(U_{\max }-v^{-}\right)}{\left(U_{c}-v^{-}\right)+\left(U_{\max }-v^{-}\right)}=R_{c r} .
$$

For the profile in figure $9, U_{\max }=0.997, U_{c}=0.594$ and according to figure 8 , $R_{c r}=-0.9$ in the inviscid limit. The resulting value of the trailing-edge velocity is then $v^{-}=0.58$ to be compared with $v^{-}=0.66$ obtained from the detailed analysis of the streak impulse response in $\S 3$. Thus, the extraction of a two-dimensional wake at the wall-normal distance of maximum Reynolds stress production is seen to lead to reasonable estimates of the trailing-edge velocity $v^{-}$, which confirms the dominant effect of the spanwise shear over its wall-normal counterpart in the triggering of the sinuous instability.

\section{Conclusions}

The main results of the present investigation may be summarized as follows: the instability of saturated optimal streaks in the flat-plate boundary layer is clearly convective. It is even more convective than the Blasius boundary layer in the absence of streaks. Whereas the trailing-edge velocity of a Tollmien-Schlichting wave packet in the Blasius boundary layer is $35 \%$ of the free-stream velocity, the trailing-edge velocity of a wave packet riding on saturated optimal streaks is around $70 \%$ of the free-stream velocity. This is because the instability of the Blasius boundary layer and of the streaks are driven by distinct physical mechanisms: the Tollimien-Schlichting instability is viscous in character and its production is located in a low-velocity region close to the wall; the streak instability is inviscid in character, it is primarily induced by the spanwise shear and its production is located further away from the wall in a higher velocity region. All the optimal streaks considered in this study are stable with respect to Tollmien-Schlichting waves, as demonstrated by Cossu \& Brandt (2002). The streak impulse response consists of the sinuous mode of instability 
triggered by the spanwise wake-like profile. This feature has been further confirmed by comparing the numerical results with the absolute/convective instability properties of the family of two-dimensional wakes constructed by Monkewitz (1988). The streak profile $U(y, z)$ may be very approximately modelled as a two-dimensional spanwise wake flow $U(z)$ which is independent of the wall-normal coordinate, provided we select a plane parallel to the wall corresponding to the peak of Reynolds stress production.

The results have been obtained under the assumption of zero-detuning $\left(\delta k_{z}=0\right)$, the flow being excited in the fundamental mode by a spanwise periodic array of localized initial perturbations with the same spanwise wavelength as the underlying streaks. A similar analysis can be conducted for the subharmonic mode $\left(\delta k_{z}=\pi / \lambda_{z}\right)$, in which case the flow is excited by a periodic array with twice the streak spanwise wavelength. Preliminary simulations indicate similar features; the subharmonic mode is also convectively unstable, with characteristic wave packet velocities that are close to those of the fundamental mode. Note that the temporal instability characteristics were also found by Andersson et al. (2001) to be insensitive to the value of the detuning parameter $\delta k_{z}$.

This study was initially motivated by the conjecture that the streak instability being essentially wakelike in the spanwise direction, it could give rise to an absolute instability for sufficiently deep profiles, as in classical wakes. Our conclusions do not confirm this conjecture; the streak instability is produced sufficiently high above the wall, in regions where local streamwise velocities are large so that perturbations are advected away. The convective nature of the secondary streak instability implies that the type of bypass transition studied here is essentially noise-driven; streaks behave as flow amplifiers in terms of the classification introduced by Huerre \& Monkewitz (1990). Intrinsic transition criteria similar to those suggested by Lingwood (1995, 1996) in rotating disk boundary layers are therefore not likely to exist. The criteria are bound to be external-noise dependent, since both the streaks and the secondary instability are noise-driven. As concerns transition in boundary layers subject to high levels of free-stream turbulence, it is important to note that the relation of the modal secondary instability to streak breakdown has not been definitely proved. Flow visualizations (Matsubara \& Alfredsson 2001) show that some streaks develop a streamwise waviness of relatively short wavelength, which these authors attribute to a secondary instability.

The present results concerning the convective nature of the instability of streaks in two-dimensional flat-plate boundary layers are in qualitative agreement with those of Koch (2002) regarding the secondary instability of secondary cross-flow vortices in three-dimensional swept-wing boundary layers. In the latter case, the impulse response wave packet pertaining to the fundamental mode (zero detuning, i.e. same periodicity as the underlying basic flow) propagates along the axis of the primary cross-flow vortices. The dominant part of the wave packet travels at around $80 \%$ of the boundary-layer edge velocity, as in the present investigation.

The near-wall dynamics of fully turbulent boundary layers is known to give rise to a self-sustaining process consisting of streamwise vortices which transfer momentum to generate streaks. In turn, the streaks are unstable and regenerate vortices (Waleffe 1995, 1997; Jeong et al. 1997; Kawahara et al. 1998; Jiménez \& Pinelli 1999; Schoppa $\&$ Hussain 2002). The instability properties of the streaky structures in wall-bounded turbulent flows are qualitatively similar to those of transitional streaks; the dominant mode is sinuous in character. In this situation, we speculate that the characteristic wave packet velocities of the regenerated vortices will be determined by the peak of 
the Reynolds stress production term. A more detailed investigation remains to be carried out.

The family of two-dimensional wake profiles introduced by Monkewitz (1988) has proved to be helpful in determining the local absolute/convective instability properties of classical wakes behind bluff bodies. The present results suggest that the analysis of a corresponding three-dimensional model $U(y, z)$ for an isolated streak would shed light on the instability characteristics of the various streaky structures encountered in transitional or fully turbulent wall-bounded flows.

The work was performed during L.B.'s visit at the Hydrodynamics Laboratory (LadHyX) and supported by École Polytechnique and TFR (Teknikvetenskapliga forskningsrådet). Computer time was provided by the Institut du Développment et des Ressources en Informatique Scientifique (IDRIS).

\section{REFERENCES}

Andersson, P., Berggren, M. \& Henningson, D. S. 1999 Optimal disturbances and bypass transition in boundary layers. Phys. Fluids 11, 134-150.

Andersson, P., Brandt, L., Bottaro, A. \& Henningson, D. S. 2001 On the breakdown of boundary layers streaks. J. Fluid Mech. 428, 29-60.

Asai, M., Minagawa, M. \& NishioKa, M. 2002 The instability and breakdown of a near-wall low-speed streak. J. Fluid Mech. 455, 289-314.

Bech, K. H., Henningson, D. S. \& Henkes, R. A. W. M. 1998 Linear and nonlinear development of localized disturbances in zero and adverse pressure gradient boundary layers. Phys. Fluids 10, 1405-1418.

BERS, A. 1983 Space-time evolution of plasma instabilities - absolute and convective. In Handbook of Plasma Physics (ed. M. N. Rosenbluth \& R. Z. Sagdeev), vol. 1, pp. 451-517. North-Holland.

Bottaro, A. \& Klingmann, B. G. B. 1996 On the linear breakdown of Görtler vortices. Eur. J. Mech. B/Fluids 15, 301-330.

Brancher, P. \& Chomaz, J. M. 1997 Absolute and convective secondary instabilities in spatially periodic shear flows. Phys. Rev. Lett. 78, 658-661.

Breuer, K. S. \& Haritonidis, J. H. 1990 The evolution of a localized disturbance in a laminar boundary layer. Part 1. Weak disturbances. J. Fluid Mech. 220, 569-594.

Brevdo, L. \& Bridges, T. 1996 Absolute and convective instability of spatially periodic flows. Phil. Trans. R. Soc. Lond. A 354, 1027-1064.

Butler, K. M. \& Farrell, B. F. 1992 Three-dimensional optimal perturbations in viscous shear flow. Phys. Fluids A 4, 1637-1650.

Chomaz, J. M., Couairon, A. \& Julien, S. 1999 Absolute and convective nature of the Eckhaus and zigzag instability with throughflow. Phys. Fluids 11, 3369-3373.

Chomaz, J. M., Huerre, P. \& Redekopp, L. G. 1991 A frequency selection criterion in spatially developing flows. Stud. Appl. Maths 84, 119-144.

Cossu, C. \& Brandt, L. 2002 Stabilization of Tollmien-Schlichting waves by finite amplitude optimal streaks in the Blasius boundary layer. Phys. Fluids 14, L57-L60.

Delbende, I. \& Chomaz, J.-M. 1998 Nonlinear convective/absolute instabilities of parallel twodimensional wakes. Phys. Fluids 10, 2724-2736.

Delbende, I., Chomaz, J.-M. \& Huerre, P. 1998 Absolute and convective instabilities in the Batchelor vortex: a numerical study of the linear impulse response. J. Fluid Mech. 355, 229-254.

Gaster, M. 1975 A theoretical model of a wave packet in a boundary layer over a flat plate. Proc. R. Soc. Lond. A 347, 271-289.

Gaster, M. \& Grant, I. 1975 An experimental investigation of the formation and development of a wave packet in a laminar boundary layer. Proc. R. Soc. Lond. A 347, 253-269.

Grosch, C. E. \& Salwen, H. 1978 The continuous spectrum of the Orr-Sommerfeld equation. Part 1. The spectrum and the eigenfunctions. J. Fluid Mech. 87, 33-54. 
Hall, P. \& Horseman, N. J. 1991 The linear inviscid secondary instability of longitudinal vortex structures in boundary layers. J. Fluid Mech. 232, 357-375.

Henningson, D. S., Lundbladh, A. \& Johansson, A. V. 1993 A mechanism for bypass transition from localized disturbances in wall-bounded shear flows. J. Fluid Mech. 250, 169-207.

HuERre, P. 1988 On the absolute/convective nature of primary and secondary instabilities. In Propagation in Systems Far from Equilibrium (ed. J. E. Wesfreid, H. R. Brand, P. Manneville, G. Albinet \& N. Boccara), pp. 340-353. Springer.

Huerre, P. 2000 Open shear flow instabilities. In Perspectives in Fluid Dynamics (ed. G. K. Batchelor, H. K. Moffatt \& M. G. Worster), pp. 159-229. Cambridge University Press.

Huerre, P. \& Monkewitz, P. A. 1990 Local and global instabilities in spatially developing flows. Annu. Rev. Fluid Mech. 22, 473-537.

Huerre, P. \& Rossi, M. 1998 Hydrodynamic instabilities in open flows. In Hydrodynamic and Nonlinear Instabilities (ed. C. Godrèche \& P. Manneville), pp. 81-294. Cambridge University Press.

Jeong, J., Hussain, F., Schoppa, W. \& Kim, J. 1997 Coherent structures near the wall in a turbulent channel flow. J. Fluid Mech. 332, 185-214.

JimÉnez, J. \& Pinelli, A. 1999 The autonomous cycle of near wall turbulence. J. Fluid Mech. 389, 335-359.

Kawahara, G., JimÉnez, J., Uhlmann, M. \& Pinelli, A. 1998 The instability of streaks in near-wall turbulence. Tech. Rep. NASA-Stanford University Centre for Turbulence Research, Annual Research Briefs, pp. 155-170.

Kim, J., Moin, P. \& Moser, R. 1987 Turbulence statistics in fully developed channel flow. J. Fluid Mech. 177, 133-166.

KocH, W. 2002 On the spatio-temporal stability of primary and secondary crossflow vortices in a three-dimensional boundary layer. J. Fluid Mech. 456, 85-112.

LeCunfF, C. \& Bottaro, A. 1993 Linear stability of shear profiles and relation to the secondary instability of the Dean flow. Phys. Fluids A 5, 2161-2171.

Lingwood, R. 1995 Absolute instability of the boundary layer on a rotating disk. J. Fluid Mech. 299, 17-33.

Lingwood, R. 1996 An experimental study of absolute instability of the rotating-disk boundarylayer flow. J. Fluid Mech. 314, 373-405.

LuCHINI, P. 2000 Reynolds-number independent instability of the boundary layer over a flat surface. Part 2. Optimal perturbations. J. Fluid Mech. 404, 289-309.

Lundbladh, A., Berlin, S., Skote, M., Hildings, C., Choi, J., Kim, J. \& Henningson, D. S. 1999 An efficient spectral method for simulation of incompressible flow over a flat plate. Tech. Rep. KTH/MEK/TR-99/11-SE. KTH, Department of Mechanics, Stockholm.

Matsubara, M. \& Alfredsson, P. H. 2001 Disturbance growth in boundary layers subjected to free stream turbulence. J. Fluid. Mech. 430, 149-168.

Monkewitz, P. 1988 The absolute and convective nature of instability in two-dimensional wakes at low Reynolds numbers. Phys. Fluids 31, 999-1006.

Morkovin, M. V. \& ReshoKTo, E. 1990 Dialogue on progress and issues in instability and transition research. In Laminar-Turbulent Transition (ed. D. Arnal \& R. Michel), pp. 3-39. Springer.

NaYFeH, A. H. \& Mook, D. T. 1979 Nonlinear Oscillations. Wiley.

Olendraru, C., Sellier, A., Rossi, M. \& Huerre, P. 1999 Inviscid instability of the Batchelor vortex: absolute-convective transition and spatial branches. Phys. Fluids 11, 1805-1820.

PARK, D. S. \& HuERre, P. 1995 Primary and secondary instabilities of the asymptotic suction boundary layer on a curved plate. J. Fluid Mech. 283, 249-272.

Pier, B. \& Huerre, P. 2001 Nonlinear self-sustained structures and fronts in spatially developing wake flows. J. Fluid Mech. 435, 145-174.

RodDier, F. 1971 Distributions et Transformation de Fourier. McGraw-Hill.

Saric, W. S., Reed, H. L. \& Kerschen, E. J. 2002 Boundary-layer receptivity to freestream disturbances. Annu. Rev. Fluid Mech. 34, 291-319.

Schlichting, H. 1979 Boundary-Layer Theory. McGraw-Hill.

Schmid, P. J. \& Henningson, D. S. 2001 Stability and Transition in Shear Flows. Springer.

Schoppa, W. \& Hussain, F. 2002 Coherent structure generation in near-wall turbulence. J. Fluid Mech. 453, 57-108. 
Swearingen, J. D. \& Blackwelder, R. F. 1987 The growth and breakdown of streamwise vortices in the presence of a wall. J. Fluid Mech. 182, 255-290.

WALEFFE, F. 1995 Hydrodynamic stability and turbulence: beyond transients to a self-sustaining process. Stud. Appl. Maths 95, 319-343.

Waleffe, F. 1997 On a self-sustaining process in shear flows. Phys. Fluids 9, 883-900.

WU, X. \& CHOUdARI, M. 2001 Linear and nonlinear instabilities of Blasius boundary layer perturbed by streamwise vortices. Part ii: Intermittent instability induced by long-wavelength Klebanoff modes. Rep. 2001-45. ICASE.

Wu, X. \& LuO, J. 2001 Instability of Blasius boundary layer in the presence of steady streaks. Annual Res. Briefs. Centre for Turbulence Research, Stanford (CA). 